\title{
INTOLERANCE IN THE MEDIA AND REPRESENTATIONS OF THE CATHOLIC CHURCH
}

\author{
David Kraner
}

Faculty of Theology

University of Ljubljana

David.Kraner@teof.uni-lj.si
UDK: 550.347.6:272]316.647.5(497.4Slovenija)

179.1:272(497.2Slovenija)

https://doi.org/10.34075/cs.56.4.8

Izvorni znanstveni rad

Rad zaprimljen 2/2021

\section{Summary}

Intolerance toward Christians in Europe including Slovenia is increasing and experts are not dealing with it sufficiently. The media plays a key role in disseminating information and shaping social representations. The fact that the media, due to the nature of their action, will always be in conflict with the Church, must not be a reason for intolerance. In Slovenia, the media are the central creators of negative opinions about the Church. They are very sophisticated in spreading Christianophobia. The journalists with the most published articles with negative connotations regarding the Church create negative social representations. Negative topics that are most often associated with the Church in the media are sexual abuse, money and politics. An analysis of the connotation and topic of the articles shows that the serious socio-political themes of the Church are neglected in the media. The local media mostly write positively about the Church, as they are aware of specific events, while the national media write about it negatively since they are often distant from specific events and usually evaluate them according to editorial policy criteria, and not according to professional arguments and varied opinions. The location of events covered negatively in articles happen both within and outside Slovenia. Most negative articles do not include photos; however, tabloids usually include them. By reducing the dissemination of negative and discriminatory messages about the Church and raising the ethics of reporting, intolerance towards Christians and other minorities will decrease.

Key words: Christianophobia, intolerance, representations, public opinion, journalism, connotation 


\section{INTRODUCTION}

In Slovenia, the number of evident cases of Christianophobia doubled in 2019 compared to the previous year. In its 2019 Annual Report, the Catholic Church in Slovenia cites six evident cases of vandalism, most of which involve the destruction of religious symbols (crosses, chapels and other buildings) and in one case an insulting article by a journalist from the Mladina magazine. ${ }^{1}$ The Observatory on Intolerance and Discrimination against Christians reports on intolerance and discrimination against Christians, which records 2,600 cases of violence against Christians that occurred in all of Europe from 2005 to 2019. Since 2015, when there were 180 such cases, ${ }^{2}$ this number has increased. In 2019, 303 cases were already recorded. ${ }^{3}$

Despite this data, there have been no new studies related to Christianophobia. Similar studies on minorities are already outdated. It is worth mentioning the study on minorities in Slovenia ${ }^{4}$ and the study on the Catholic Church and the media owned by the Church. ${ }^{5}$ In the whole of Europe, however, there is a very interesting study on the demonized representations of Muslims. ${ }^{6}$ However, this study does not directly address the aforementioned issues.

Given that the number of cases of intolerance and discrimination against Christians in Europe is increasing, this will have to be more widely addressed in the future. In this article, we wish to examine this issue in Slovenia in more detail from the point of view of the media and their representations of the Catholic Church. Since it is the media that promotes the "circulation" of information among people and, therefore, form and strengthen individual and social representations, we have to prioritize them, as the results of the study will also show other aspects of the issue of Christianophobia.

1 Secretariat SŠK, Letno poročilo 2019, November 22 2019, https:/ / katoliska-cerkev. si/media/datoteke/Dokumenti\%20in\%20publikacije/LETNO\%2OPOROCILO\%20 2019-OBJAVA\%20SPLET.pdf (acquired December 1 2019), 154-160.

2 Ellen Fantini, Report 2018, Observatory on Intolerance and Discrimination against Christians in Europe, Vienna, 2018, 19.

3 Ellen Fantini, Report 2019, Observatory on Intolerance and Discrimination against Christians in Europe, Vienna, 2019, 29.

4 Petković et al., Spremljanje in vrednotenje medijev. Anketa med študenti, etničnimi manjšinami in politiki $v$ Sloveniji, Mirovni inštitut, Ljubljana, 2009.

5 Sandra B. Hrvatin - Brankica Petković, In temu pravite medijski trg? Vloga države $v$ medijskem sektorju $v$ Sloveniji, Mirovni inštitut, Ljubljana, 2007.

$6 \quad$ Paul Baker et al., Discourse Analysis and Media Attitude. The Representation of Islam in the British Press, Cambridge University Press, Cambridge, 2013; Saied R. Ameli et al., The British Media and Muslim Representation: The Ideology of Demonisatio. Islamic human Rights commission, Wembley, 2007. 


\section{Social Representations, the Media and the Church}

The media encourages rapid dissemination of information among people. Social and individual representations depend on this fluctuating dynamic. The functioning of the Church is different in nature from the media, and this creates conflicts between the media and the Church. The problem arises when the media do not do their job and become active participants in the spread of Christianophobia, instead of the truth.

According to Cardinal Dulles, the media and the Church differ in at least seven different areas. 1) The Church has content at its disposal with a mysterious message about God and the redemptive presence of Jesus Christ in the world. The media, however, with their investigative nature of breaking down tradition, is far from respectful and enjoy imaginative, inaccurate and scandalous explanations. 2) The fundamental message of the Church is the Gospel, which is eternally valid; therefore, it seeks continuity with the past and thus maintains its stability. In contrast, the media are interested in discoveries and make a living from novelty. 3) The Church strives to spread unity, reconciliation and conflict resolution. The media, which are experts in creating tensions and conflicts, evidently create the greatest possible interest and dissemination of information. 4) The Church speaks of invisible grace in the light of eternal salvation, while the media speak exclusively of tangible phenomena. 5) The Church is a hierarchical organization, its leaders act in the name of Christ's authority and apostolic succession. The media cannot accept such an arrangement because they are subject only to the democratic evaluation criteria of any organization. For them, a disobedient priest and a controversial theologian are a great example of freedom. 6) The Church is very complex in its religious teaching and moral practices and always close to a misunderstanding so it is natural and necessary to use a language of complex technical terms, dogmas and morals in these sensitive matters. The language is too difficult for an ordinary person to understand. The media, however, use short and simple storied that grab attention. 7) The Church wishes to convince its listeners about the truth of revelation, which requires a thorough personal commitment of an individual to his personal faith and the proclamation of Christ. The media, however, report on events that are also acceptable to infidels. ${ }^{7}$

7 Avery Dulles, Religion and the News Media: A Theologian Reflects, in: America, 9 (1994), 6-9. 
In the issue of Christianophobia, a person is essential in addition to the Church and the media. Social representations (SR) are more important than public opinion. Sergej Moscovici, the founder of the theory of social representations, defines them as a set of theories and ideologies, connected in the interaction of persons of a society or group. Their characteristic is that they transform (transmute) individual ideas into collective experiences, and interactions into collective behaviours. Social representations originate from the modern society, the political, scientific, and humane environment. They are dynamic, as rapid changes in society do not allow them to form into something permanent and unchanging. SRs directly increase heterogeneity in society and change "unification systems" (of official sciences, religions, ideologies). Where they cause changes, they become collective realities. The mass media accelerate the emergence of SRs by fostering heterogeneity in society and further multiplying changes and creating the need for connections between completely abstract "sciences" (or collective beliefs on one hand) and concrete activities of social individuals on the other. ${ }^{8}$

Connecting the individual and collective is also very important for Stuart Hall, who emphasizes the importance of speech. The connection between thought concepts and speech allows us a connection to real objects, persons and events and to imaginary worlds, objects, persons and events. There are two processes of representations. First, an individual system that connects a whole combination of objects, persons, and events with a series of thought concepts or representations that exist in our heads: for example our concepts of war, death, friendship or love. The conceptual system alone is not sufficient. It is necessary to exchange, that is, to represent meanings and thought concepts. We can do this with a language that is common (shared) to all. The global process of constructing speech is the other representative system. Our individual concept maps need to be shared with others, they need to be translated into a common language so that we can relate them to the concepts and ideas of others. Words, voices and images are signs. These signs represent concepts and the relationships between them. ${ }^{9}$

Furthermore, some scientists emphasize that in representations the line between the individual and the collective is blurred. Among these, the social psychologist, Mirjana Ule, argues that individual representations (IRs) of objects, persons, events, and ide-

$8 \quad$ Serge Moscovici, Le rappresentazioni sociali, Mulino, Bologna, 2013, 29.

$9 \quad$ Hall et al., Representation, The Open University SAGE, London, 2013, 3-5. 
as (reality) are difficult to distinguish from social representations (SRs). People use SR, but they are also their creators. Communities in our society would not be able to function without social representations. ${ }^{10}$ Ule also emphasizes the importance of the circulation of SR among people and emphasizes, "that [SRs] coexist both in the human mind as part of his cognitive apparatus, and at the same time circulate among people as parts of intersubjective reality. They are reflected in the ways of speech, discourse, jargon in media messages, in manuals, instructions for use and popular literature. In social representations, they support each other subjectively and objectively in a way, one cannot exist without the other. Therefore, the processes of subjective cognition as an individual representation of reality necessarily presuppose the functioning of social representations. Individual representations are subjective, naive, or implicit theories of man about different areas of reality. Social representations, however, represent a communal, social framework for individual thinking and knowledge as well as for the social division of knowledge". ${ }^{11}$

The circulation of SR among people is best taken care of by the media. The basic journalistic rule says that bad news is good news for a newspaper editorial office and that bad influence is good influence for media research, because it is the subject of research in which it is possible to gain greater visibility in public opinion, political and social responsibility, radio and television stations. ${ }^{12}$ However, this is not good for people's SRs: negative SRs cause a negative attitude. Negative SRs are also an expression of negative internal attitude or behaviour of the individual.

The task of journalists is to inform the public with news and the truth. News that contains elements of Christianophobia is often incorrect and thereby distorts the truth. The media thus inserts the truth "into the wrong context and thus leads people to a conclusion that is wrong but useful for it". ${ }^{13}$ Because readers cannot know when the media is not providing them with the real facts, they generally accept any information as the truth. There is a kind of unwritten contract or pact between the media and readers. The Italian social

10 Mirjana Ule, Socialna psihologija. Analitični pristop $k$ življenju $v$ družbi, Založba FDV, Ljubljana, 2009, 113.

11 Ule, Socialna psihologija, 112.

12 Gianni Losito, Il potere dei madia, Carocci, Rome, 2007, 116.

13 Peter Lah, Laži, preoblječne $v$ novice, da ljudje dobimo norice, (February 15 2018), www.academia.edu/35927042/Fake_news_Laži_preoblečene_v_novice _ ki_povzročijo_da_ljudje_dobimo_norice (acquired September 9 2020), 1. 
psychologist Ida Galli believes that people "accept any news that is reported by the media, whether it is the press, radio or television, on the assumption that the information is true, even though it is in fact erroneous, as it was communicated by a person who is in the service of the truth. This is essential as it connects the audience with their media source. Although it may happen that the news is later revoked and a correction (denial) is published, even though contradictory statements are published and even though all the evidence shows something completely different, this information has gained the validity of the truth that it was communicated and understood in the light of the said pact". ${ }^{14}$

Today, the mass media is losing their power, and personal media is coming to the fore. ${ }^{15}$ Individuals follow only the topics they are interested in, therefore, their condition of being informed is shrinking according to the topics and sources they follow. The narrow condition of being informed reduces the need for critical thinking and the demand for quality information, leaving the leading media free to choose and create leading information topics. In addition, we cannot ignore the claim of sociologist Mauel Castels, who believes that the greatest power belongs to the media who are supported by the social bearers of power. ${ }^{16}$ In Slovenia, the most influential televisions in the field of news programs are national TV Slovenia and commercial Pop TV; among the newspapers, the most influential are Večer and Dnevnik. ${ }^{17}$ Our study shows that these media also convey the most negative information about the Catholic Church. This indicates the fact that in Slovenia those in power (political and economic) fail to perform their duty when it comes to preventing Christianophobia.

Our view of the world through the eyes of the media is not transparent. The problem of opacity that journalists introduce into public discourse will remain forever. ${ }^{18}$ In this way, the SRs will also constantly change as directed by media managers. Fake and Christi-

14 Ida Galli, La teoria delle rappresentazioni sociali, Il Mulino, Bologna, 2006, 78-79.

15 Marco Pratellesi, New Journalism. Teorie e tecniche del giornalismo multimediale, Bruno Mondadori, Milan, 2008, 206.

16 Manuel Castells, Comunicazione e Potere, Università Bocconi Editore, Milano, 2009, 137.

17 P. A, Najbolj priljubljeni slovenski mediji v 2018: Nedeljski dnevnik, Radio 1, Pop TV and 24ur.com. (March 27 2019), https://moski.hudo.com/aktualno/najbolj-priljubljeni-slovenski-mediji-v-2018-nedeljski-dnevnik-radio-1-pop-tv-24urcom/ (acquired September 9 2020).

18 Massimo Russo - Zambardino Vittorio, Eretici digitali. La rete è in pericolo, il giornalismo pure come salvarsi con un tradimento e 10 tesi, Apogeo, Milan, 2009, 128. 
anophobic news is negative. As such, they are much more influential in changing attitudes, internal attitudes, and even the behaviour of individuals in society. Even those who do not follow the media receive information the media through their family members, friends or acquaintances. Lazarsfeld's theory of two-stage media influence, although seventy years old, is therefore still relevant: the media does not have a direct influence on the individual, but is influenced indirectly through opinion leaders. ${ }^{19}$ Due to this influence, the media have the potential to trigger the change of positive SRs in the direction of negative SRs with negative news. This is happening with the SR on the Catholic Church.

In short, SRs are not an indisputable reality about reality, but something that is created based on what happens in the environment. If the environment is negative and poisoned, then so will be the social representations and vice versa.

\section{TEXT ANALYSIS OF ARTICLES}

We have analysed 3735 articles of print, radio, television and online media in four trimestral analytical periods in 2019. Kliping d.o.o., a company for monitoring and analysis of media, daily provided us with the articles in which the Catholic Church was mentioned in. Relevant articles were analysed with an analytical scheme, a questionnaire in an Excel document. Quantitative analyses were performed with the SPSS program, and qualitative analyses with the T-LAB program. The analysis includes newspaper articles, radio and television reports of the news program and online news of the Slovenian Press Agency (STA). Different types of texts are hereinafter referred to as 'articles', although they are radio or TV reports. To facilitate data processing, we have grouped local media into the following categories: "Other-Print", "Other-Radio", "Other-TV" and "Other-Web".

The question we wished to obtain an answer to was how the Slovenian media represent the Catholic Church. We presumed that their representations of the Catholic Church have the potential to influence the social representations people have about the Catholic Church. Our analytical scheme: a questionnaire was prepared based on obtaining answers to the following research questions: Who pro-

19 Elihu Katz - Paul F. Lazarsfeld, L'influenza personale in comunicazione, Armando, Rome, 2012, 47-48. 
duces information about the Catholic Church? What is their content? When do they publish? Where do they publish? How do they represent the content? The following categories emerge from these issues: authors, topics, publication periods, media, and connotation of content and photographs.

\subsection{Overview of categories throughout the four periods}

The analytical scheme was evaluated from period to period in order to obtain the most accurate answers to the research questions. Thus, the number of questions from the first to the third period was optimized according to the content of the articles. The first period, which contains articles from January 1 to March 31, 2019, comprises 48 analytical questions. The articles from this period, unlike other periods, are analysed in more detail. The analytical scheme also includes characteristics of an article (hierarchy, length), venue, title (which institutional name is used for the Church) and persons (which ecclesiastical persons are presented in the article) as well as the appearance of political figures or other celebrities in connection with the Catholic church). The analysis of the second period of articles, from April 1 to June 31 2019, comprised only 16 analytical questions. What is new in this period is a detailed analysis of the venue, the type of contribution and the connotation of the pictorial material. The analysis of the third period, between July 1 and September 30,2019 , is the shortest and contains only 6 analytical questions: identification number, medium, author of the article, topic, connotation of image and text. To the fourth period, between October 1 and December 31 2019, only the category type of media text (genre) was added to the categories of the previous period.

\section{MonovarianT ANALYSES}

In this part, we will present the analysed media, authors, topics, connotations of the texts and the presence of photographs in the texts.

\subsection{Media}

The first period includes 1246 articles. The number of articles in this period is higher than in other periods, as we also analysed the articles that did not directly concern the Catholic Church. In this 
way, we allowed an analyses of those articles that concern religion, religiosity, religions, Islam, terrorism, etc. The largest percentage of all analysed articles were published in local media, "Other-print"20, the online media sta.si, Dnevnik and Delo.

Table 1. Number of analysed articles in each period according to each media

\begin{tabular}{lrrrr}
\hline N & Period 1 & Period 2 & Period 3 & Period 4 \\
Delo & 114 & 42 & 60 & 102 \\
Demokracija & 38 & 15 & 32 & 28 \\
Dnevnik & 125 & 75 & 78 & 97 \\
Other - Radio & 13 & 15 & 21 & 20 \\
Other - Print & 268 & 63 & 235 & 337 \\
Other- TV & 49 & 38 & 24 & 43 \\
Mladina & 35 & 16 & 20 & 18 \\
Radio Slovenija & 104 & 28 & 50 & 64 \\
Reporter & 34 & 20 & 36 & 24 \\
Slovenske novice & 56 & 34 & 73 & 76 \\
Sta.si & 176 & 65 & 83 & 155 \\
Svet24 & 34 & 31 & 21 & 28 \\
TV Slovenija & 106 & 34 & 49 & 67 \\
Večer & 94 & 30 & 52 & 80 \\
Other-Web & 0 & 10 & 0 & 0 \\
Total & 1246 & 516 & 834 & 1139 \\
& & & Total: & 3735 \\
\hline
\end{tabular}

In the second period, 516 articles were analysed, two thirds less than in the first period. As mentioned, the criteria for selecting articles for analysis were re-evaluated: during this period, only those articles directly describing the Catholic Church were analysed, and all those describing Islam, Orthodoxy, Protestantism, or other religions were omitted, as the Catholic Church was not mentioned. Short

20 In the first period, in the category of local press, the following print media were included in "print-other“: Bistriške novice, Celjan, Dolenjski list, Domžalske novice, Domžalski slamnik, Gorenjski glas - Jeseniške novice, Gorenjski Glas, Idrijske novice, Kmečki Glas, Koroška, Listi Šoštanja, Novi tednik Celje, Posavski dnevnik, Posavski obzornik, Prepih, Primorske novice, Primorski dnevnik, Rogaške novice, Šentjurčan, Štajerc, Štajerski Tednik, Utrip Savinjske doline, Vestnik Murska Sobota. 
articles, briefly mentioning a local event and mentioning the Church in passing were also omitted. Most articles, analysed from this period, were from sta.si, Dnevnik, Slovenske novice and "print-other" 21.

In the third period, 834 articles were analysed. Unlike the second period, the criteria for selecting articles also included articles regarding parish celebrations that were excluded in the second period. During this period, most articles were again published in the "Other-print" 22 , followed by sta.si, Dnevnik, Slovenske novice and Delo.

In the fourth period, 1139 articles were analysed. The selection criteria were the same as in the third period and proved to be the best. In this period, most articles were again in "Other-print" ${ }^{23}$, in Sta.si, Delo, Dnevnik and Večer.

From Table 1 The number of analysed articles in each period according to media, it is evident that the number of analysed articles in the four periods varies considerably. In the first period, all articles in which the church, religion and religiosity were mentioned were analysed, even if they were not directly related to the Catholic Church. In the second period, only those articles that exclusively described the Catholic Church were analysed. Articles related to any topic (including non-ecclesiastical) and local events mentioning the Catholic Church were also included in the third and fourth periods. Numerical difference between the first, the third and the fourth period, indicates that Sta.si, Delo, Dnevnik, Mladina, Radio Slovenia, TV Slovenia and Večer report on religious topics almost as much as they do on the Catholic Church: the numbers halve in the 3rd and 4 th periods. However, there are no such differences in "Other-print" (or local press), "Other-TV", Reporter and Demokracija.

21 In the second period, the following print media were included in the "Other-print" category: Avenija, Delauska enotnost, Domovina, Global, Gorenjski glas, Kmečki glas, Manager, Madrač, Naš čas, Novi tednik Celje, Primorske novice, Primorski dnevnik and Sensa.

22 In the third period, the following print media were included in the "Otherprint” category: Bistriške novice, Bukla, Domžalske novice, Domžalski slamnik, Družina, Ekipa, Finance, Glas gospodarstva, Global, Idrijske novice, Koroška, Krošnjar, Lady, Liza, Manager, Naš čas, National Geographic, Novi glas, Novice slovenske konjice, Posavski obzornik, Obrazi, Oko, Panorama, Posavski dnevnik, Pravna praksa, Prepih, Rogaške novice, Savinjske novice, SGlasnik, Stop, Suzy, Šentjurčan, Štajerc, Štajerski tednik, Utrip Savinjske doline, Vzajemna, Zarja, Zeleni raj and Ženska.

23 The print media from the first three periods were included in the category of local press, "Other-print" in the fourth period. In the analysis, we did not keep a list of articles for individual local media. 


\subsection{Author}

Authors of articles, journalistic subjects (journalists, photographers, editors, persons involved in journalistic discourse) create media reality by choosing, asking, summarizing, processing, changing, narrating information. ${ }^{24}$ Media houses evaluate and reward performances according to who has the greater amount of information, and is first and original in reporting. As this is very difficult for burning topics, they use shortcuts to unofficially obtain information from an 'insider'. In terms of the "church" topic, the most interesting people for journalists are lay people, priests and bishops through whom they receive confidential information. Successful journalists ensure their appearance in the media and in the public, and thus their trust with people. "A person with a piece of information will give it to the public media or a journalist who enjoys the greatest reputation and trust in the media space. /.../ People prefer to trust those journalists whom they consider to be their "defenders', and are less open to those whom they consider to be part of the establishment." 25 Furthermore, journalists must first follow the policy of their media, which has established criteria for reporting on individual topics, institutions and persons.

In 2019, 1107 different authors wrote about the Catholic Church, journalists, correspondents, experts in individual fields, other individuals (letters from readers) and church dignitaries (bishops, priests, religious). It was not possible to determine the authorship of 530 articles by Sta.si, 585 articles without signatures and 74 authors who used only initials. 647 authors wrote one article, 208 authors two articles, 83 authors three and 169 authors four or more (Table 2.).

24 Melita Polner Kovačič, Novinarska (iz)virnost. Novinarji in njihovi viri $v$ sodobni slovenski družbi, Fakulteta za družbene vede, Ljubljana, 2004, 23-24.

25 Matjaž Šuen, Preiskovalno novinarstvo, Fakulteta za družbene vede, Ljubljana, 1994, 30. 
Table 2. Authors with the greatest number of articles

\begin{tabular}{|c|c|c|c|c|c|c|c|c|}
\hline & Medium & $\mathrm{N}$ & & Medium & $\mathrm{N}$ & & Medium & $\mathrm{N}$ \\
\hline 1 & $\begin{array}{l}\text { Editorial } \\
\text { offices }\end{array}$ & 590 & 58 & $\begin{array}{l}\text { Miranda } \\
\text { Bratkič }\end{array}$ & 7 & 115 & $\begin{array}{l}\text { Uroš } \\
\text { Pušnik }\end{array}$ & 5 \\
\hline 2 & Sta.si & 530 & 59 & $\begin{array}{l}\text { Nuša } \\
\text { Lesar }\end{array}$ & 7 & 116 & $\begin{array}{l}\text { Žiga } \\
\text { Pohleven }\end{array}$ & 5 \\
\hline 3 & $\begin{array}{l}\text { Janko } \\
\text { Petrovec }\end{array}$ & 85 & 60 & $\begin{array}{l}\text { Peter } \\
\text { Štumpf }\end{array}$ & 7 & 117 & $\begin{array}{l}\text { Aleks } \\
\text { Horvat }\end{array}$ & 4 \\
\hline 4 & $\begin{array}{l}\text { Jože } \\
\text { Košnjek }\end{array}$ & 37 & 61 & $\begin{array}{l}\text { Petra } \\
\text { Kerčmar }\end{array}$ & 7 & 118 & $\begin{array}{l}\text { Alenka } \\
\text { Sivka }\end{array}$ & 4 \\
\hline 5 & $\begin{array}{l}\text { Ranka } \\
\text { Ivelja }\end{array}$ & 31 & 62 & $\begin{array}{l}\text { Rosvita } \\
\text { Pesek }\end{array}$ & 7 & 119 & Ali Žerdin & 4 \\
\hline 6 & $\begin{array}{l}\text { Janez } \\
\text { Markež }\end{array}$ & 26 & 63 & $\begin{array}{l}\text { Sonja Gri- } \\
\text { zila }\end{array}$ & 7 & 120 & $\begin{array}{l}\text { Andraž } \\
\text { Sodja }\end{array}$ & 4 \\
\hline 7 & $\begin{array}{l}\text { Peter } \\
\text { Petrovčič }\end{array}$ & 26 & 64 & Uros Slak & 7 & 121 & $\begin{array}{l}\text { Anja } \\
\text { Intihar }\end{array}$ & 4 \\
\hline 8 & $\begin{array}{l}\text { Oste Bakal } \\
\text { (O.B.) }\end{array}$ & 24 & 65 & $\begin{array}{l}\text { Urša } \\
\text { Peternel }\end{array}$ & 7 & 122 & $\begin{array}{l}\text { Anja } \\
\text { Vučkič }\end{array}$ & 4 \\
\hline 9 & $\begin{array}{l}\text { Lidija Markelj } \\
\text { (L.M.) }\end{array}$ & 24 & 66 & $\begin{array}{l}\text { Barbara } \\
\text { Hočevar }\end{array}$ & 6 & 123 & Apa (6) & 4 \\
\hline 10 & Ivan Puc & 19 & 67 & $\begin{array}{l}\text { Brane } \\
\text { Jeranko }\end{array}$ & 6 & 124 & Beb (7) & 4 \\
\hline 11 & Jan Novak & 18 & 68 & $\begin{array}{l}\text { Dejan } \\
\text { Kovac }\end{array}$ & 6 & 125 & $\begin{array}{l}\text { Bernarda } \\
\text { Jeklin }\end{array}$ & 4 \\
\hline 12 & Jožica Grgič & 18 & 69 & $\begin{array}{l}\text { Dejan } \\
\text { Ladika }\end{array}$ & 6 & 126 & $\begin{array}{l}\text { Biljana } \\
\text { Polak }\end{array}$ & 4 \\
\hline 13 & Tatjana Pihlar & 18 & 70 & G.B. (4) & 6 & 127 & $\mathrm{BJ}(8)$ & 4 \\
\hline 14 & Vlado Began & 18 & 71 & $\begin{array}{l}\text { Igor } \\
\text { Bergant }\end{array}$ & 6 & 128 & C.R. (9) & 4 \\
\hline 15 & $\begin{array}{l}\text { A. Nana Ritu- } \\
\text { per Rodež }\end{array}$ & 18 & 72 & $\begin{array}{l}\text { Igor } \\
\text { Mravlja }\end{array}$ & 6 & 129 & $\begin{array}{l}\text { Cirila } \\
\text { Sever }\end{array}$ & 4 \\
\hline 16 & Nataša Lang & 16 & 73 & $\begin{array}{l}\text { Marjan } \\
\text { Horvat }\end{array}$ & 6 & 130 & cost (10) & 4 \\
\hline 17 & $\mathrm{dr}(1)$ & 15 & 74 & $\begin{array}{l}\text { Mirjam } \\
\text { Muženic }\end{array}$ & 6 & 131 & $\begin{array}{l}\text { Helena } \\
\text { Florenin } \\
\text { Pasinato }\end{array}$ & 4 \\
\hline 18 & $\begin{array}{l}\text { Urška } \\
\text { Mlinarič }\end{array}$ & 15 & 75 & $\begin{array}{l}\text { Mojca } \\
\text { Zemljarič }\end{array}$ & 6 & 132 & Igor Gošte & 4 \\
\hline 19 & Barbara Štor & 14 & 76 & Rok Kuželj & 6 & 133 & J.K. (11) & 4 \\
\hline
\end{tabular}




\begin{tabular}{|c|c|c|c|c|c|c|c|}
\hline 20 & Lucija Kavčič & 14 & 77 & $\begin{array}{l}\text { Sara } \\
\text { Lotrič }\end{array}$ & 6 & 134 & $\begin{array}{l}\text { Jana } \\
\text { Krebelj }\end{array}$ \\
\hline 21 & Mihael Šorl & 14 & 78 & $\begin{array}{l}\text { Sebastjan } \\
\text { Valentan }\end{array}$ & 6 & 135 & $\begin{array}{l}\text { Jaroslav } \\
\text { Jankovič }\end{array}$ \\
\hline 22 & $\begin{array}{l}\text { Uroš Škerl } \\
\text { Kramberger }\end{array}$ & 14 & 79 & $\begin{array}{l}\text { Vili } \\
\text { Einspieler }\end{array}$ & 6 & 136 & $\begin{array}{l}\text { Jože } \\
\text { Gabor }\end{array}$ \\
\hline 23 & $\begin{array}{l}\text { Biserka } \\
\text { Karneža }\end{array}$ & 13 & 80 & $\begin{array}{l}\text { Zorana } \\
\text { Baković }\end{array}$ & 6 & 137 & $\begin{array}{l}\text { Jože } \\
\text { Miklave }\end{array}$ \\
\hline 24 & $\begin{array}{l}\text { Elen Batista } \\
\text { Štader }\end{array}$ & 13 & 81 & $\begin{array}{l}\text { Aleksan- } \\
\text { der Lucu }\end{array}$ & 5 & 138 & $\begin{array}{l}\text { Jure } \\
\text { Trampuš }\end{array}$ \\
\hline 25 & $\begin{array}{l}\text { Gašper } \\
\text { Blažič }\end{array}$ & 13 & 82 & $\begin{array}{l}\text { Alenka } \\
\text { Arko }\end{array}$ & 5 & 139 & $\begin{array}{l}\text { Katja Kirn } \\
\text { Vodopivec }\end{array}$ \\
\hline 26 & Primož Hieng & 13 & 83 & $\begin{array}{l}\text { Alenka } \\
\text { Tratnik }\end{array}$ & 5 & 140 & $\begin{array}{l}\text { Lara } \\
\text { Paukovic }\end{array}$ \\
\hline 27 & $\begin{array}{l}\text { Danica Zavrl } \\
\text { Žlebir }\end{array}$ & 12 & 84 & $\begin{array}{l}\text { Andreja } \\
\text { Gregorič }\end{array}$ & 5 & 141 & Lidija Kosi \\
\hline 28 & Vasja Jager & 11 & 85 & Anka Pirš & 5 & 142 & $\begin{array}{l}\text { Luka } \\
\text { Bregar }\end{array}$ \\
\hline 29 & $\begin{array}{l}\text { Karmen W. } \\
\text { Švegl }\end{array}$ & 10 & 86 & $\begin{array}{l}\text { Branko } \\
\text { Cestnik }\end{array}$ & 5 & 143 & $\begin{array}{l}\text { Maja } \\
\text { Bertoncelj }\end{array}$ \\
\hline 30 & $\begin{array}{l}\text { Magični } \\
\text { gledalec }\end{array}$ & 10 & 87 & Franci Kek & 5 & 144 & $\begin{array}{l}\text { Manca } \\
\text { Turk }\end{array}$ \\
\hline 31 & Miha Naglič & 10 & 88 & $\begin{array}{l}\text { Gordana } \\
\text { Stojiljko- } \\
\text { vić }\end{array}$ & 5 & 145 & $\begin{array}{l}\text { Marjeta } \\
\text { Klemenc }\end{array}$ \\
\hline 32 & Aleš Žužek & 9 & 89 & Ines Baler & 5 & 146 & $\begin{array}{l}\text { Marta } \\
\text { Razboršek }\end{array}$ \\
\hline 33 & $\begin{array}{l}\text { Marjana } \\
\text { Ahačič }\end{array}$ & 9 & 90 & $\begin{array}{l}\text { Ivan } \\
\text { Štuhec }\end{array}$ & 5 & 147 & $\begin{array}{l}\text { Meta } \\
\text { Černoga }\end{array}$ \\
\hline 34 & Peter Colnar & 9 & 91 & $\begin{array}{l}\text { Janez } \\
\text { Kuhar }\end{array}$ & 5 & 148 & $\begin{array}{l}\text { Miha } \\
\text { Pogačnik }\end{array}$ \\
\hline 35 & $\begin{array}{l}\text { Svetlana } \\
\text { Slapšak }\end{array}$ & 9 & 92 & $\begin{array}{l}\text { Jasna } \\
\text { Krljić Vreg }\end{array}$ & 5 & 149 & $\begin{array}{l}\text { Mihael } \\
\text { Korsika }\end{array}$ \\
\hline 36 & Anita Kribiš & 8 & 93 & $\begin{array}{l}\text { Jasna } \\
\text { Paladin }\end{array}$ & 5 & 150 & $\begin{array}{l}\text { Miloš } \\
\text { Šonc }\end{array}$ \\
\hline 37 & Janko Votek & 8 & 94 & $\begin{array}{l}\text { Jernej } \\
\text { Suhadol- } \\
\text { nik }\end{array}$ & 5 & 151 & $\begin{array}{l}\text { Miro } \\
\text { Štebe }\end{array}$ \\
\hline 38 & $\begin{array}{l}\text { Katarina } \\
\text { Braniselj }\end{array}$ & 8 & 95 & $\begin{array}{l}\text { Lovro } \\
\text { Kastelic }\end{array}$ & 5 & 152 & $\begin{array}{l}\text { Mojca } \\
\text { Hanžilč }\end{array}$ \\
\hline
\end{tabular}




\begin{tabular}{|c|c|c|c|c|c|c|c|c|}
\hline 39 & $\begin{array}{l}\text { Katarina } \\
\text { Golob }\end{array}$ & 8 & 96 & $\begin{array}{l}\text { Luka } \\
\text { Potočnik }\end{array}$ & 5 & 153 & N.K. (12) & 4 \\
\hline 40 & $\begin{array}{l}\text { Marcel } \\
\text { Štefančič }\end{array}$ & 8 & 97 & $\begin{array}{l}\text { Luka } \\
\text { Tetičković }\end{array}$ & 5 & 154 & N.N. (13) & 4 \\
\hline 41 & $\begin{array}{l}\text { Marko } \\
\text { Medvešek }\end{array}$ & 8 & 98 & $\begin{array}{l}\text { Majda } \\
\text { Horvat }\end{array}$ & 5 & 155 & $\begin{array}{l}\text { Nenad } \\
\text { Glücks }\end{array}$ & 4 \\
\hline 42 & $\begin{array}{l}\text { Polona } \\
\text { Jamnik }\end{array}$ & 8 & 99 & $\begin{array}{l}\text { Marina } \\
\text { Vrbnjak }\end{array}$ & 5 & 156 & pk (14) & 4 \\
\hline 43 & Saša Krajnc & 8 & 100 & $\begin{array}{l}\text { Maša } \\
\text { Likosar }\end{array}$ & 5 & 157 & $\begin{array}{l}\text { Sara } \\
\text { Horžen }\end{array}$ & 4 \\
\hline 44 & $\begin{array}{l}\text { Suzana P. } \\
\text { Kovačič }\end{array}$ & 8 & 101 & $\begin{array}{l}\text { Metod } \\
\text { Berlec }\end{array}$ & 5 & 158 & $\begin{array}{l}\text { Senka } \\
\text { Dreu }\end{array}$ & 4 \\
\hline 45 & $\begin{array}{l}\text { Tomaž } \\
\text { Klipšteter }\end{array}$ & 8 & 102 & Mgć (5) & 5 & 159 & $\begin{array}{l}\text { Spomenka } \\
\text { Hribar }\end{array}$ & 4 \\
\hline 46 & $\begin{array}{l}\text { Toni } \\
\text { Jurjevec }\end{array}$ & 8 & 103 & $\begin{array}{l}\text { Milan } \\
\text { Glavonjić }\end{array}$ & 5 & 160 & $\begin{array}{l}\text { Stanislav } \\
\text { Zore }\end{array}$ & 4 \\
\hline 47 & $\begin{array}{l}\text { Vanessa } \\
\text { Čokl }\end{array}$ & 8 & 104 & $\begin{array}{l}\text { Monika } \\
\text { Horvat }\end{array}$ & 5 & 161 & $\begin{array}{l}\text { Staša } \\
\text { Lozar }\end{array}$ & 4 \\
\hline 48 & D.N. (2) & 7 & 105 & $\begin{array}{l}\text { Nina } \\
\text { Mrzlikar }\end{array}$ & 5 & 162 & $\begin{array}{l}\text { Tatjana } \\
\text { Popovič }\end{array}$ & 4 \\
\hline 49 & Darja Zgonc & 7 & 106 & Peter Rak & 5 & 163 & U.S. (15) & 4 \\
\hline 50 & $\begin{array}{l}\text { Dejan } \\
\text { Vodovnik }\end{array}$ & 7 & 107 & $\begin{array}{l}\text { Rok } \\
\text { Valenčič }\end{array}$ & 5 & 164 & $\begin{array}{l}\text { Vesna } \\
\text { Humar }\end{array}$ & 4 \\
\hline 51 & $\begin{array}{l}\text { Drago Karl } \\
\text { Ocvirk }\end{array}$ & 7 & 108 & $\begin{array}{l}\text { Simon } \\
\text { Šubic }\end{array}$ & 5 & 165 & $\begin{array}{l}\text { Vesna } \\
\text { Levicnik }\end{array}$ & 4 \\
\hline 52 & $\begin{array}{l}\text { Gregor } \\
\text { Trebušak }\end{array}$ & 7 & 109 & T. Tavčar & 5 & 166 & $\begin{array}{l}\text { Vlado } \\
\text { Miheljak }\end{array}$ & 4 \\
\hline 53 & J.Z. (3) & 7 & 110 & $\begin{array}{l}\text { Tatjana } \\
\text { Vrbnjak }\end{array}$ & 5 & 167 & $\begin{array}{l}\text { Vojislav } \\
\text { Brecko }\end{array}$ & 4 \\
\hline 54 & $\begin{array}{l}\text { Janez } \\
\text { Turinek }\end{array}$ & 7 & 111 & $\begin{array}{l}\text { Tina Hor- } \\
\text { vat }\end{array}$ & 5 & 168 & $\begin{array}{l}\text { Živa } \\
\text { Slaobir }\end{array}$ & 4 \\
\hline 55 & $\begin{array}{l}\text { Jasmina } \\
\text { Jamnik }\end{array}$ & 7 & 112 & $\begin{array}{l}\text { Tomaž } \\
\text { Ranc }\end{array}$ & 5 & 169 & $\begin{array}{l}\text { Zvezdana } \\
\text { Bercko }\end{array}$ & 4 \\
\hline 56 & $\begin{array}{l}\text { Marjana } \\
\text { Debevec }\end{array}$ & 7 & 113 & $\begin{array}{l}\text { Tone } \\
\text { Petelinšek }\end{array}$ & 5 & & $\begin{array}{l}\text { Other } \\
(\mathrm{N}<3)\end{array}$ & 1310 \\
\hline 57 & Mateja Rant & 7 & 114 & $\begin{array}{l}\text { Uroš } \\
\text { Badovinac }\end{array}$ & 5 & & Total & 3735 \\
\hline
\end{tabular}


Texts, published by the media are not transparent or neutral, but strongly conditioned by editorial policy. From the connotation of the articles, the editorial policy of a particular medium is evident. In this study, we can highlight only those who published 15 or more articles in 2019 and were included in our analysis (Table 3. Comparison of the most productive authors by periods). There are 16 such authors. With their frequent write-ups about the Church, they create and disseminate public opinion about the Catholic Church.

The number before the parentheses indicates the number of articles they wrote, and from the numbers in parentheses it is evident, the opinion they create and disseminate about the Church. They represent the connotation of the articles: the first number represents the number of articles with positive connotation, the second with negative connotation, the third general (both positive and negative) and the fourth ideological connotation. For example, of the total 85 analysed articles from Janko Petrovec, a RTV Slovenija correspondent, 49 articles have a positive, 8 negative, 6 general (positive and negative) and 22 have ideological connotation.

To sum up the analysis of the authors, we can claim that there are some authors on the national (TV and radio) and local media who report on the Church most positively. Among them are: Jože Košnjek, Lidija Markelj, "dr" and Janko Petrovec. There are more authors who always write negatively about the Church. These are: Vlado Began, Urška Mlinarič, Ranka Ivelja, Peter Petrovčič, Janez Markež and Jožica Grgič. The greatest number of ideological articles can be found in Ranka Ivelja in Dnevnik. The conclusion is that in Slovenia we have the greatest number of authors who write negatively about the Church; that we have only one journalist on national television who reports positively about the Church, that authors who write articles with negative connotations work for Večer, Delo, Dnevnik and Mladina, and that journalists in the local media usually write articles about the Church with a positive connotation.

\subsection{Topics related to the Catholic Church}

Topics, in the analysed articles, related to the Catholic Church, were grouped into main categories for further analysis: 1) money and property, 2) charity, 3) ethical issues - euthanasia, 4) abortion and cloning, 5) topics, related to migration, 6) post-war massacres, 7) political topics, 8) feasts and celebrations, 9) religious topics and religious dialogue, 10) topics on education, 11) hate speech and 
Table 3. Comparison of the most productive authors in each period

\begin{tabular}{|c|c|c|c|c|c|c|}
\hline & Author & Period 1 & Period 2 & Period 3 & Period 4 & TOTAL \\
\hline 1 & $\begin{array}{l}\text { Janko } \\
\text { Petrovec }\end{array}$ & $\begin{array}{l}41 \\
(16,5,1,19)\end{array}$ & $\begin{array}{l}14 \\
(11,0,2,1)\end{array}$ & $\begin{array}{l}7 \\
(6,0,0,1)\end{array}$ & $\begin{array}{l}23 \\
(16,3,3,1)\end{array}$ & $\begin{array}{l}85 \\
(49,8,6,22)\end{array}$ \\
\hline 2 & $\begin{array}{l}\text { Jože } \\
\text { Košnjek }\end{array}$ & $9(9,0,0,0)$ & $\begin{array}{l}6 \\
(2,1,0,3)\end{array}$ & $\begin{array}{l}7 \\
(6,0,1,0)\end{array}$ & $\begin{array}{l}15 \\
(11,0,0,4)\end{array}$ & $\begin{array}{l}37 \\
(28,1,1,7)\end{array}$ \\
\hline 3 & $\begin{array}{l}\text { Ranka } \\
\text { Ivelja }\end{array}$ & $7(0,4,1,2)$ & $\begin{array}{l}11 \\
(0,6,4,1)\end{array}$ & $\begin{array}{l}4 \\
(1,0,3,0)\end{array}$ & $\begin{array}{l}9 \\
(3,3,2,1)\end{array}$ & $\begin{array}{l}31 \\
(4,13,10,4)\end{array}$ \\
\hline 4 & $\begin{array}{l}\text { Janez } \\
\text { Markež }\end{array}$ & $\begin{array}{l}13 \\
(1,7,4,1)\end{array}$ & $\begin{array}{l}5 \\
(0,2,3,0)\end{array}$ & $\begin{array}{l}3 \\
(0,2,1,0)\end{array}$ & $\begin{array}{l}5 \\
(0,3,0,2)\end{array}$ & $\begin{array}{l}26 \\
(1,14,8,3)\end{array}$ \\
\hline 5 & $\begin{array}{l}\text { Peter } \\
\text { Petrovčič }\end{array}$ & $\begin{array}{l}10 \\
(1,6,3,0)\end{array}$ & $\begin{array}{l}7 \\
(0,5,1,1)\end{array}$ & $\begin{array}{l}3 \\
(0,2,0,1)\end{array}$ & $\begin{array}{l}6 \\
(0,4,0,2)\end{array}$ & $\begin{array}{l}26 \\
(1,17,4,4)\end{array}$ \\
\hline 6 & $\begin{array}{l}\text { Oste } \\
\text { Bakal }\end{array}$ & $4(4,0,0,0)$ & $\begin{array}{l}5 \\
(1,1,2,1)\end{array}$ & $\begin{array}{l}6 \\
(2,3,0,1)\end{array}$ & $\begin{array}{l}9 \\
(7,1,0,1)\end{array}$ & $\begin{array}{l}24 \\
(14,5,2,3)\end{array}$ \\
\hline 7 & $\begin{array}{l}\text { Lidija } \\
\text { Markelj } \\
\text { (L.M.) }\end{array}$ & $9(7,0,2,0)$ & $\begin{array}{l}0 \\
(0,0,0,0)\end{array}$ & $\begin{array}{l}8 \\
(8,0,0,0)\end{array}$ & $\begin{array}{l}7 \\
(6,0,0,1)\end{array}$ & $\begin{array}{l}24 \\
(21,0,2,1)\end{array}$ \\
\hline 8 & Ivan Puc & $5(1,1,1,2)$ & $\begin{array}{l}3 \\
(1,0,2,0)\end{array}$ & $\begin{array}{l}7 \\
(1,0,0,6)\end{array}$ & $\begin{array}{l}4 \\
(2,0,0,2)\end{array}$ & $\begin{array}{l}19 \\
(5,1,3,10)\end{array}$ \\
\hline 9 & $\begin{array}{l}\text { Jan } \\
\text { Novak }\end{array}$ & $5(1,3,0,1)$ & $\begin{array}{l}3 \\
(1,1,1,0)\end{array}$ & $\begin{array}{l}1 \\
(1,0,0,0)\end{array}$ & $\begin{array}{l}9 \\
(6,2,0,1)\end{array}$ & $\begin{array}{l}18 \\
(9,6,1,2)\end{array}$ \\
\hline 10 & $\begin{array}{l}\text { Tatjana } \\
\text { Pihlar }\end{array}$ & $7(3,4,0,0)$ & $\begin{array}{l}1 \\
(0,0,0,1)\end{array}$ & $\begin{array}{l}0 \\
(0,0,0,0)\end{array}$ & $\begin{array}{l}10 \\
(3,1,0,6)\end{array}$ & $\begin{array}{l}18 \\
(6,5,0,7)\end{array}$ \\
\hline 11 & $\begin{array}{l}\text { Jožica } \\
\text { Grgič }\end{array}$ & $9(1,6,1,1)$ & $\begin{array}{l}2 \\
(0,1,1,0)\end{array}$ & $\begin{array}{l}3 \\
(3,0,0,0)\end{array}$ & $\begin{array}{l}4 \\
(1,2,1,0)\end{array}$ & $\begin{array}{l}18 \\
(5,9,3,1)\end{array}$ \\
\hline 12 & $\begin{array}{l}\text { Vlado } \\
\text { Began }\end{array}$ & $\begin{array}{l}10 \\
(0,10,0,0)\end{array}$ & $\begin{array}{l}2 \\
(0,2,0,0)\end{array}$ & $\begin{array}{l}1 \\
(0,1,0,0)\end{array}$ & $\begin{array}{l}5 \\
(0,5,0,0)\end{array}$ & $\begin{array}{l}18 \\
(0,18,0,0)\end{array}$ \\
\hline 13 & $\begin{array}{l}\text { A. Nana } \\
\text { Rituper } \\
\text { Rodež }\end{array}$ & $5(0,3,1,1)$ & $\begin{array}{l}1 \\
(0,0,1,0)\end{array}$ & $\begin{array}{l}7 \\
(5,1,0,1)\end{array}$ & $\begin{array}{l}5 \\
(1,0,0,4)\end{array}$ & $\begin{array}{l}18 \\
(6,4,2,6)\end{array}$ \\
\hline 14 & $\begin{array}{l}\text { Nataša } \\
\text { Lang }\end{array}$ & $5(0,0,0,5)$ & $\begin{array}{l}2 \\
(0,1,1,0)\end{array}$ & $\begin{array}{l}5 \\
(4,0,1,0)\end{array}$ & $\begin{array}{l}4 \\
(2,0,0,2)\end{array}$ & $\begin{array}{l}16 \\
(6,1,2,7)\end{array}$ \\
\hline 15 & $\mathrm{dr}$ & $6(2,1,2,1)$ & $\begin{array}{l}1 \\
(1,0,0,0)\end{array}$ & $\begin{array}{l}2 \\
(2,0,0,0)\end{array}$ & $\begin{array}{l}6 \\
(4,0,0,2)\end{array}$ & $\begin{array}{l}15 \\
(9,1,2,3)\end{array}$ \\
\hline \multirow[t]{2}{*}{16} & $\begin{array}{l}\text { Urška } \\
\text { Mlinarič }\end{array}$ & $2(1,1,0,0)$ & $\begin{array}{l}3 \\
(0,2,0,1)\end{array}$ & $\begin{array}{l}4 \\
(2,2,0,0)\end{array}$ & $\begin{array}{l}6 \\
(3,1,2,0)\end{array}$ & $\begin{array}{l}15 \\
(6,6,2,1)\end{array}$ \\
\hline & Total & 147 & 66 & 68 & 127 & 408 \\
\hline
\end{tabular}


riots, 12) sexual abuse, 13) terrorism, 14) art and culture, 15) historical themes, and 16) other. ${ }^{26}$

The articles from the first period were analysed by determining the presence of all topics in each article: if the author of an article addressed several topics, we recorded all of them (Table 4. Number and percentage of articles by topic related to the Catholic Church in the first period). This choice did not turn out to be the best: it prevented us from looking at the central theme of the article and the final proportion of articles by topic for a particular period. Therefore, in the following periods, we identified only the central topics of each article, which at the same time had to be related to the Catholic Church (Table 5. Number and share of articles according to the topics related to the Catholic Church). The topic that stood out the most in the first period was Church celebrations. This theme probability stands out because after the $1^{\text {st }}$ of January, there are many celebrations associated with the celebration of Christmas (Catholic and Orthodox) and with traditional blessings and customs (Saint Blaise's blessing, blessing of horses, blessing of wine, etc.). That is followed by evergreen topics that are popular with journalists: sexual abuse, politics, money and terrorism.

In the other three periods, where in each article we chose only the main topic related to the Church, you can clearly see the proportion of articles regarding the topic in each period. The number of events, celebrations in the second period is lower: during this period events are the least reported, and we also excluded from our analysis short articles that mention the Church only indirectly. Interestingly, the proportion of these articles increases in the third and fourth periods. In the summer of the third period, when journalists lack topics and the Church celebrates August 15, the Assumption of Mary, the proportion of articles increases. The topic of sexual abuse is quite common in the first and second periods. From the meeting of the presidents of the bishop's conferences from all over the world and those responsible in religious communities, which took place from February 21 to 242019 in Rome until April16, when the Notre Dame Church in Paris burned, the topic of sexual abuse

26 A comparative view of different periods requires that the topics that have been broken down in individual periods be grouped into one category so that they are mutually comparable. Different topics are grouped under the label "other": the first period covers the topics "Orthodoxy", "Islam", "Protestantism", "Judaism", "Islam", "Buddhism", "Adventists", "Pentecostals" and "other religions". ; the second period covers "affair - current" and "other"; the third period covers "affair" and "other" and the fourth covers "other", "renovation and restoration", "pilgrimage", "doctrine of the RCC", "sexuality" and "music". 
Table 4. Number and percentage of articles related to the Catholic Church in the first period

\begin{tabular}{rlrcrr}
\hline & Period 1 & $\mathrm{N}$ & \% Present & \% Absent & $\mathrm{N}$ \\
\hline 1 & Event & 327 & 26,2 & 73,8 & 327 \\
2 & Sexual abuse & 278 & 22,3 & 77,7 & 278 \\
3 & Politcs & 178 & 14,3 & 85,7 & 178 \\
4 & Orthodoxy & 105 & 8,4 & 91,6 & 105 \\
5 & Islam & 85 & 6,8 & 93,2 & 85 \\
6 History & 82 & 6,6 & 93,4 & 82 \\
7 & Charity & 57 & 4,6 & 95,4 & 57 \\
8 & Ethics (euthanasia, & 46 & 3,7 & 96,3 & 46 \\
& abortion) & & & & \\
9 & Hate speech - riots & 43 & 3,5 & 96,5 & 43 \\
10 & Migrants & 39 & 3,1 & 96,9 & 39 \\
11 & Art & 39 & 3,1 & 96,9 & 39 \\
12 & School system & 35 & 2,8 & 97,2 & 35 \\
13 & Afterwar massacres & 29 & 2,3 & 97,7 & 29 \\
14 & Terrorism & 29 & 2,3 & 97,7 & 29 \\
15 & Doctrine of RKC & 20 & 1,6 & 98,4 & 20 \\
16 & Protestantism & 11 & 0,9 & 99,1 & 11 \\
17 & Judaism & 5 & 0,4 & 99,6 & 5 \\
18 & Islam & 3 & 0,2 & 99,8 & 3 \\
19 & Budism & 3 & 0,2 & 99,8 & 3 \\
20 & Adventists & 0,2 & 99,8 & 2 \\
21 & Other religions & 0,2 & 99,8 & 2 \\
22 & The Pentecostal & & 99,9 & 1 \\
& Church & & & \\
\hline & & & & \\
\hline
\end{tabular}

was represented daily in media. Until the fourth period, when the civil initiative Dovolj.je revives this topic in Slovenia, this topic does not appear in the media. The next topic the media strongly associates the Church with is politics: in the first and last periods, the association of politics with the Church is present. The topic money stands out the most in the fourth period. The topic charity of is also present. The media associate charity with Caritas Slovenia and present it separately from the Church. There are also topics concerning history, migrants, ethics, religious dialogue, art, educational system and history. 
Table 5. Number and percentage of articles according to the main topics related to the Catholic Church

\begin{tabular}{|c|c|c|c|c|c|c|c|c|c|}
\hline & Period 2 & $\mathrm{~N}$ & $\%$ & Period 3 & $\mathrm{~N}$ & $\%$ & Period 4 & $\mathrm{~N}$ & $\%$ \\
\hline 1 & $\begin{array}{l}\text { Sexual } \\
\text { abuse }\end{array}$ & 143 & 27,7 & Events & 429 & 51,4 & $\begin{array}{l}\text { Celebrations } \\
\text { (events) }\end{array}$ & 287 & 25,2 \\
\hline 2 & Event & 88 & 17,1 & Politics & 63 & 7,6 & Politics & 148 & 13 \\
\hline 3 & Afairs & 69 & 13,4 & Money & 53 & 6,4 & Money & 126 & 11,1 \\
\hline 4 & Politics & 43 & 8,3 & Afairs & 48 & 5,8 & Charity & 124 & 10,9 \\
\hline 5 & Money & 39 & 7,6 & $\begin{array}{l}\text { School } \\
\text { system }\end{array}$ & 43 & 5,2 & Culture & 80 & 7 \\
\hline 6 & Terrorism & 17 & 3,3 & Charity & 38 & 4,6 & $\begin{array}{l}\text { Sexual abu- } \\
\text { se }\end{array}$ & 60 & 5,3 \\
\hline 7 & History & 16 & 3,1 & History & 33 & 4 & History & 51 & 4,5 \\
\hline 8 & $\begin{array}{l}\text { Ethics } \\
\text { (euthanasia, } \\
\text { abortion) }\end{array}$ & 13 & 2,5 & $\begin{array}{l}\text { Sexual } \\
\text { abuse }\end{array}$ & 31 & 3,7 & $\begin{array}{l}\text { Renovatins } \\
\text { and restora- } \\
\text { tions }\end{array}$ & 42 & 3,7 \\
\hline 9 & $\begin{array}{l}\text { School } \\
\text { system }\end{array}$ & 10 & 1,9 & Migrants & 25 & 3 & Pilgrimage & 38 & 3,3 \\
\hline 10 & Migrants & 9 & 1,7 & $\begin{array}{l}\text { Ethics } \\
\text { (euthana- } \\
\text { sia, abor- } \\
\text { tion) }\end{array}$ & 20 & 2,4 & $\begin{array}{l}\text { Doctrine of } \\
\text { RKC }\end{array}$ & 35 & 3,1 \\
\hline 11 & $\begin{array}{l}\text { Afterwar } \\
\text { massacres }\end{array}$ & 9 & 1,7 & Art & 14 & 1,7 & Sex & 22 & 1,9 \\
\hline 12 & Charity & 7 & 1,4 & Massacres & 8 & 1 & $\begin{array}{l}\text { School } \\
\text { system }\end{array}$ & 15 & 1,3 \\
\hline 13 & Art & 5 & 1 & Religions & 7 & 0,8 & $\begin{array}{l}\text { Hate speech } \\
\text { - riots }\end{array}$ & 14 & 1,2 \\
\hline 14 & $\begin{array}{l}\text { Hate } \\
\text { speech - } \\
\text { riots }\end{array}$ & 3 & 0,6 & $\begin{array}{l}\text { Hate } \\
\text { speech - } \\
\text { riots }\end{array}$ & 5 & 0,6 & Migrants & 10 & 0,9 \\
\hline 15 & Religions & 1 & 0,2 & Terrorism & 2 & 0,2 & Art & 9 & 0,8 \\
\hline 16 & / & & & Absent & 11 & 1,3 & $\begin{array}{l}\text { Religions- } \\
\text { dialogue }\end{array}$ & 9 & 0,8 \\
\hline 17 & / & & & / & & & $\begin{array}{l}\text { Ethics } \\
\text { (euthanasia, } \\
\text { abortion) }\end{array}$ & 9 & 0,8 \\
\hline 18 & / & & & / & & & Music & 5 & 0,4 \\
\hline \multirow[t]{2}{*}{19} & Other & 44 & 8,5 & Other & 15 & 1,6 & Other & 55 & 4,8 \\
\hline & $\begin{array}{c}\text { Total } \\
\text { period } 2\end{array}$ & 516 & 100 & $\begin{array}{c}\text { Total } \\
\text { period } 3\end{array}$ & 834 & 100 & $\begin{array}{c}\text { Total } \\
\text { period } 4\end{array}$ & 1139 & 100 \\
\hline
\end{tabular}


The category "hate speech - riots" is present in 43 articles in the first period, is almost non-existent in the second and third, and seven articles appear again in the fourth period. Most of them are related to the Secretary-General of the Slovenian Bishops' Conference, Fr. Tadej Strehovec. The case has its roots at the beginning of 2016. As the editor of the blog 24kul.si, Strehovec allowed the publication of the article List of members of the abortion lobby against the right to life, which promotes the execution of unborn girls and boys! On March 4, 2019, the court acquitted him of these convictions. Just before that, in November 2018, he was threatened with death. One of the graffiti in Ljubljana reads, "Tadej Strehovec, you will be dead." This story clearly shows that in Slovenia it is not allowed to have an opinion that is contrary to the opinions spread in the media. T. Strehovec resisted this and risked deprivation of liberty and his own life. He himself sees this as a deprivation of fundamental rights to religious freedom. In Noelle-Neumann's eyes, however, this is a typical example of the media effect of the spiral of silence.27 Intimidating Christians from practicing their faith in public is also evidenced by an empirical study of the Social Representation of the Catholic Church in Slovenia, where in eight focus groups participants highlight the fear of being Catholics in college, at work or elsewhere in public. ${ }^{28}$

In conclusion, we can claim that the Church is associated in the media with fourteen main topics, and most often with topics on sexual abuse, politics, money and parish events-celebrations. Although Christianophobia is not one of the most present topics, it is nevertheless strongly present when it comes to politically motivated events. Therefore, most of the articles on this topic are from the first period when Fr. Strehovec was put to court, are almost absent in the second and third periods, but reappears in the fourth. Indications of Christianophobia can be found not only as a topic of "hate speech" but also in the connotation of texts that subtly spread hostile messages about the Church. We have presented the authors in cross-reference to the number and connotation of articles, now we will present the connotation of articles.

27 Elisabeth Noelle-Neumann, La spirale del silenzio. Per una teoria dell'opinione pubblica, MELTEMI, Rome, 2002, 18-21.

28 David Kraner, Le rappresentazioni sociali della Chiesa Cattolica in Slovenia, LAS, Rome, 2019, 198. 


\subsection{Connotation of articles by periods}

The connotation of a text has a strong influence on the co-creation of public opinion. Professor Zannachi is convinced that "public opinion is the result of a process in which communication is essential. Public opinion is a psychosocial phenomenon that consists of a mental study (individual psychological aspect), within an audience or a group (subjective aspect), in connection with facts and problems of a public nature (objective aspect), which is formed through interactions that operate within a collective (social aspect) and can be objectively measured with tools adapted to it. Public opinion can remain unexpressed, it can be measured with appropriate tools (surveys) and it can be expressed through behaviour (manifestation, appeal, voting). In short, if public opinion is based on democratic participation, on the exchange of arguments of a public nature, it can assume the function of social control." 29

Public opinion co-creates images and social representations that allow people to feel better in their environment and communicate more easily with others. "The formation of public opinion is largely tied to an individual's ability to form information, compose mental representations and mental structures or mental schemes that serve to interpret and control their environment". ${ }^{30}$ When public opinion is untrue, so are the images and social representations of a particular institution or topic.

For the analysis, the texts were evaluated based on four different connotations: positive, negative, general (both positive and negative) and ideological (related to interests). The criterion for an article with positive connotation requires that the text describes the Catholic Church as entirely positive. Such articles prevail in all four periods (Graph 1. Connotation of texts in all four periods by percentage): In the third and fourth periods, it exceeds by half, in the first and second by a third of all published articles. A text with negative connotation describes the Catholic Church only negatively. The largest number of articles with negative connotation can be found in the second period, and the lowest number in the third. The comprehensive connotation of a text is the one that emphasizes the positive and negative descriptions of the Catholic Church in the same article. The highest number of articles is in the second period, and the lowest in the fourth. All the articles that had neither positive nor

29 Adriano Zanacchi, Opinione pubblica, mass media, propaganda, Las, Rome, 2006, 124-125.

30 Vincent Price, L'opinione pubblica, Il Mulino, Bologna, 2004, 66. 
negative descriptions of the Church, but only general, indifferent or ideological content were labelled "ideological". The contents of these articles are often inaccurate and related to the interests of certain social groups, as the authors of such articles represent either an organization, institution, party, ideology (home guards, right-left political parties, partisans) or topic (education system, taxes, protection of minors) which directly or indirectly affects the Catholic Church. This also includes articles that use the word "church" and their subject of discourse are those institutions, buildings that used to be church buildings, but not anymore (e.g. the phrases "church holding", "križanke", "Baraga seminary", etc.). The highest number of articles with ideological connotation can be found in the fourth and first periods.

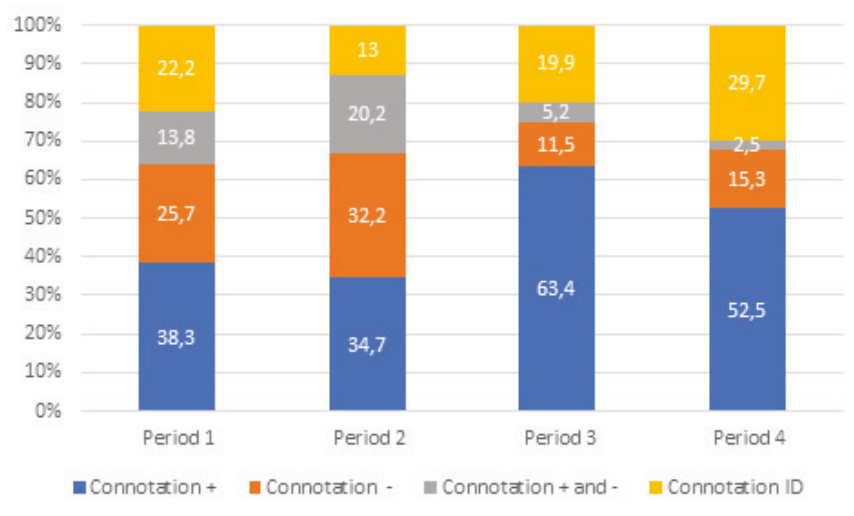

Graph 1. Connotation of articles in all four periods by percentage

Research proves that the media influences individuals by strengthening their opinion, reshaping it or creating an entirely new opinion. Their influence is also possible on an individual's inner attitudes, standpoint, and behaviour. ${ }^{31}$ In addition, it should be pointed out that the media can also influence people who do not follow the media through the two-stage media influence, where the media directly influences the "leaders", persons who are protagonists in a certain field and are exposed to the media, who then influence the

31 Joseph T. Klaper, The Effects of Mass Communication, Free Press, Glencoe Illinois, 1960; Denis McQuail, Mass Communicatiion Theory an introduction, Sage, London, 1983; Mauro Wolf, Teorie delle comunicazioni di massa, Bompini, Milan, 1992. 
people around them with their opinion. ${ }^{32}$ Among other things, the media is very careful not to go beyond the critical limit with negative reporting on the Church that could cause a boomerang effect. ${ }^{33}$

Table 6. Connotation of articles by periods.

\begin{tabular}{|llllllllllll}
\hline $\begin{array}{l}\text { Konot. } \\
\text { Ideol }\end{array}$ & $\begin{array}{l}\text { Peri- } \\
\text { od 1 }\end{array}$ & $\%$ & $\begin{array}{l}\text { Peri- } \\
\text { od 2 }\end{array}$ & $\%$ & $\begin{array}{l}\text { Peri- } \\
\text { od 3 }\end{array}$ & $\%$ & $\begin{array}{l}\text { Peri- } \\
\text { od } 4\end{array}$ & $\%$ & Total & $\%$ \\
1 & $\begin{array}{l}\text { Connota- } \\
\text { tion + }\end{array}$ & 477 & 38,3 & 179 & 34,7 & 529 & 63,4 & 598 & 52,5 & 1783 & 47,7 \\
2 & $\begin{array}{l}\text { Connota- } \\
\text { tion - }\end{array}$ & 320 & 25,7 & 166 & 32,2 & 96 & 11,5 & 174 & 15,3 & 756 & 20,3 \\
3 & $\begin{array}{l}\text { Conno- } \\
\text { tation + } \\
\text { and - }\end{array}$ & 277 & 22,2 & 104 & 20,2 & 43 & 5,2 & 29 & 2,5 & 348 & 9,3 \\
\hline $\begin{array}{l}\text { Connota- } \\
\text { tion ID }\end{array}$ & 172 & 13,8 & 67 & 13 & 166 & 19,9 & 338 & 29,7 & 848 & 22,7 \\
\hline & 1246 & 100 & 516 & 100 & 834 & 100 & 1139 & 100 & 3735 & 100 \\
\hline
\end{tabular}

From the analysis on the comparison of the connotation of articles between periods, it is evident that a total of almost half of the articles convey a positive connotation. About $43 \%$ of the articles convey an ideological and negative connotation (Table 6. Connotation of articles by periods). Most of these articles are persuasive in structure and with clear intention to influence readers. Hovland emphasizes that readers of such texts find themselves faced with a decision whether to insist on an opinion that is contrary to the opinion of the media and completely reject it, or accept it and thus change the inner attitude to the topic or content reported by the media. He adds that even information with very low credibility can have a positive effect due to the long-term reporting to the addressee. ${ }^{34}$ Although news with negative and ideological connotation is most popular with readers (Barbano 2012, 30-31), they have a very negative impact on them. ${ }^{35}$

Among the articles that report directly or indirectly on the Church, almost half carry a negative and ideological connotation. Since

32 Paul Lazarsfeld et. al., Voting. A study of opinion formation in a presidential campaign, University of Chicago Press, Chicago, 1954.

33 Gianni Losito, Il potere dei madia, Carocci, Rome, 2007, 88-89.

34 Gianni Losito, Il potere dei madia, Carocci, Rome, 2007, 95-97.

35 Gianni Losito, Il potere del pubblico. La fruizione dei mezzi di comunicazione di massa, Carocci, Rome, 2003, 59. 
news with a negative and ideological connotation in the long run is likely to have a negative impact on public opinion about the Church and consequently on the internal attitude, standpoint and behaviour of readers, we can conclude that the Slovenian media is in favour of Christianophobia. However, there are very few articles with a comprehensive connotation (14\% of articles), in which authors present comprehensive opinion with positive and negative arguments. Such reporting does not educate people to be responsible citizens and is therefore most alarming.

\subsection{The media and connotation of the article}

The power of the media depends on many factors that change periodically. The effectiveness of campaigns and the power of the media are limited and conditioned by an individual sphere, the political system, the cultural system, general practices, the electoral system, possible transitions and crisis situations. ${ }^{36}$ The church as a part of society is integrated in these systems and is therefore often criticized by the media. This is evident from the connotation of their articles.

The largest number of articles with positive connotations are found in the local press, and the largest number of articles with negative connotation is found in Dnevnik, Delo and Sta.si (Table 7. Connotation of articles by media). The highest number of articles with general connotation was recorded in Dnevnik, Delo, Radio Slovenia, TV Slovenia and Sta.si., articles with ideological connotation in the local press in Sta.si, Dnevnik and Delo. When we look at the total number of analysed articles, those with positive and ideological connotations stand out the most. It is interesting that in Delo and Dnevnik there are such articles in connection with the negative connotation, and in the local press and Sta.si with the positive one.

In the analysed articles, among the very critical and negative attitudes towards the Catholic Church, it is expected that everyone agree with the prescribed public opinion. The individual opinions of influential people are strongly asserted through the Slovenian media as the only true ones. The ideological and negative connotations of the articles increasingly spread fear among dissenters and lock them in a spiral of silence. ${ }^{37}$

36 Gianni Losito, Il potere dei madia, Carocci, Rome, 2007, 101.

37 Elisabeth Noelle-Neumann, La spirale del silenzio. Per una teoria dell'opinione pubblica, MELTEMI, Rome, 2002, 18-21. 
Table 7. Connotation of articles by media

\begin{tabular}{|c|c|c|c|c|c|c|}
\hline & & $\begin{array}{l}\text { Conno- } \\
\text { tation+ }\end{array}$ & $\begin{array}{l}\text { Conno- } \\
\text { tation - }\end{array}$ & $\begin{array}{l}\text { Conno- } \\
\text { tation + } \\
\text { and - }\end{array}$ & $\begin{array}{l}\text { Conno- } \\
\text { tation } \\
\text { ID }\end{array}$ & Total \\
\hline 1 & Delo & 90 & 101 & 39 & 88 & 318 \\
\hline 2 & Demokracija & 46 & 13 & 16 & 38 & 113 \\
\hline 3 & Dnevnik & 96 & 141 & 46 & 92 & 375 \\
\hline 4 & Other - Radio & 47 & 8 & 2 & 12 & 69 \\
\hline 5 & Other - Print & 584 & 95 & 35 & 189 & 903 \\
\hline 6 & Other - TV & 63 & 38 & 26 & 27 & 154 \\
\hline 7 & Mladina & 6 & 53 & 8 & 22 & 89 \\
\hline 8 & Radio Slovenija & 146 & 32 & 37 & 31 & 246 \\
\hline 9 & Reporter & 41 & 15 & 20 & 38 & 114 \\
\hline 10 & Slovenske novice & 127 & 44 & 15 & 53 & 239 \\
\hline 11 & Sta.si & 251 & 67 & 35 & 126 & 479 \\
\hline 12 & Svet24 & 34 & 39 & 18 & 23 & 114 \\
\hline 13 & TV Slovenija & 136 & 43 & 33 & 44 & 256 \\
\hline 14 & Večer & 113 & 61 & 17 & 65 & 256 \\
\hline \multirow[t]{2}{*}{15} & Web & 3 & 6 & 1 & 0 & 10 \\
\hline & Total & 1783 & 756 & 348 & 848 & 3735 \\
\hline
\end{tabular}

To conclude, we can claim that the local media write and report on the Church mostly with a positive connotation because they are in direct contact with the local Church (priest and parish) and because they report on specific events. Dnevnik, Delo and some other media write and report on the Church with a negative connotation because they have no contact with specific events in the Catholic Church, with people, while adhering more to their editorial policy than to the truthfulness of events.

\subsection{Presence of photographs in articles}

For many readers alluring photos are decisive when choosing whether they will read an article. Photographs are considered a more direct way of communicating as opposed to texts, and represent the dynamics of power. "Photography, as far as being a tool of journalism and at the same time a medium in which society accepts the additional property of reality and objectivity, constructs virtual forms of its own realism and its own objectivity in relation to the defi- 
nitions of "relevance" defined by various media, consumer products in their competition in relation to the market." ${ }^{38}$ According to a very good marketing analysis on their effectiveness, they are the most attractive for readers or viewers besides the title. Despite the fact that people are "able to accurately distinguish between an idea and the reality of a thing, as we have the ability to move through concept or image from an idea to reality of an object" 39 , we are often misled by false and untrue media images. Walter Lippman, a journalist and a scientist who studied public opinion, argues, "Human behaviour is constantly determined by how the world around them is presented to them" 40 . The environment represented by untrue and subjective images can eventually become true. For example, hostile texts discriminating against minorities, can become a source of violence.

There are almost $70 \%$ of such articles with photographs about the Church. The tabloids Slovenske novice and Svet24 have the highest number of articles with photographs (between 85 and 100\%). Delo and Dnevnik, which are more central, have the lowest number of photographs (Table 8. Presence of photographs in articles). When comparing the data on the presence of photographs with the connotation of articles according to the media (Table 7. Connotation of articles according to the media) we can see that Delo and Dnevnik, which contain articles with the most negative connotation, have a lower number of articles with photos compared to other media. Local media, Demokracija, Slovenske novice and Svet24 have more articles with photographs. The prevailing connotation of articles in these media is not the same everywhere, as in Demokracija and Slovenske novice the positive and ideological connotations prevail, and in Svet24 articles with negative connotations prevail.

In conclusion, we can assert that the media that report negatively on the Church do not as a rule provide articles with photographs. The tabloids Slovenske novice and Svet24 have a greater number of articles with photographs. Since the national media have less contact with specific events, the number of their articles about the Church has fewer photographs than the local media.

38 Andrea Pogliano, Le immagini delle notizie. Sociologia del fotogiornalismo, Edizioni Unicopli, Milan, 20092009, 26.

39 Serge Moscovici, Le rappresentazioni sociali, Mulino, Bologna, 2013, 9.

40 Walter Lippmann, Public Opinion, Harcourt - Brace, New York, 1922, 212. 
Table 8. Presence of photographs in articles

\begin{tabular}{lllllllll}
\hline N & $\begin{array}{l}\text { Peri- } \\
\text { od } 1\end{array}$ & $\%$ & $\begin{array}{l}\text { Peri- } \\
\text { od } 2\end{array}$ & $\%$ & $\begin{array}{l}\text { Peri- } \\
\text { od } 3\end{array}$ & & $\begin{array}{l}\text { Peri- } \\
\text { od } 4\end{array}$ & $\%$ \\
Delo & 89 & 78,1 & 31 & 73,8 & 40 & 66,7 & 76 & 74,5 \\
Demokracija & 34 & 89,5 & 14 & 93,3 & 31 & 96,9 & 23 & 82,1 \\
Dnevnik & 82 & 65,6 & 48 & 64 & 52 & 66,7 & 69 & 71,1 \\
Other - Radio & 0 & 0 & 0 & 0 & 0 & 0 & 0 & 0 \\
Other - print & 217 & 81 & 47 & 74,6 & 204 & 86,8 & 286 & 84,9 \\
Other - TV & 49 & 100 & 38 & 100 & 24 & 100 & 43 & 100 \\
Mladina & 30 & 85,7 & 14 & 87,5 & 18 & 90 & 16 & 88,9 \\
Radio & 0 & 0 & 0 & 0 & 0 & 0 & 0 & 0 \\
Slovenija & & & & & & & & \\
Reporter & 26 & 76,5 & 18 & 90 & 32 & 88,9 & 21 & 87,5 \\
Slovenske & 48 & 85,7 & 30 & 88,23 & 68 & 93,2 & 75 & 98,7 \\
novice & & & & & & & & \\
Sta.si & 0 & 0 & 0 & 0 & 0 & 0 & 0 & 0 \\
Svet24 & 31 & 91,2 & 25 & 80,65 & 20 & 95,2 & 24 & 100 \\
TV Slovenija & 106 & 100 & 34 & 100 & 49 & 100 & 67 & 100 \\
Večer & 83 & 88,30 & 24 & 80 & 45 & 86,5 & 68 & 85 \\
Other-Web & 0 & 0 & 5 & 5 & 0 & 0 & 0 & 0 \\
\hline Total & 795 & 63,8 & 319 & 61,82 & 583 & 69,9 & 772 & 67,8 \\
All articles & 1246 & 100 & 516 & 100 & 834 & 100 & 1139 & 100 \\
All periods & & & & & & & 3735 & 100 \\
\hline
\end{tabular}

\section{BIVARIATE ANALYSES}

According to the British sociologist Stuart Hall, known for his coding and decoding communication model and studies on how the media represent news, consumers can understand media content in several ways. Regarding the coding, multimedia messages are first produced, expanded afterwards and finally interpreted. Regarding decoding, however, three different approaches emerge: reflective, intentional, and constructionist. In the reflective approach, the meaning lies in an object, a person, an idea or an event in the real world where language functions like a mirror to reflect the true meaning that already exists in the world. Although we can fully understand words, voices and images, there is a risk that this description 
can only be a fantasy or subjective imagination. In the intentional approach, the path to understanding the meaning is reversed. An author gives meaning to an object, a person, an idea or an event. The author extracts from words, voices and images what he wished them to mean. Consequently, people constantly need to coordinate with each other, as their words, voices, or images that appear in speech constantly change meaning. The third, the constructionist approach, emphasizes the public social dimension of language. It establishes that neither things per se (reflective approach) nor authors (intentional approach) can fix meaning in a language. Things by themselves do not have meaning; the people who decode them within a community or culture give meaning to them. Hence, it is called a constructionist approach. ${ }^{41}$

Among the essential features of the news is the proximity of the event: whether something occurred within my proximity and concerns me as well, or whether it happened in a distant place and does not concern me. In this regard, we were interested in whether the negative reporting on the Church was related to foreign countries or as they occurred in Slovenia. In the following points, bivariate analyses from two areas are presented: 1) connotations and location, 2) connotations and topics of articles.

\subsection{Connotations and location}

In the first and second periods ${ }^{42}$, the study also included the location of an individual article: Slovenia, abroad, Slovenia and abroad at the same time and the absence of data on the location (Graph 2. Connotation of article according to location in the 1st and 2nd periods). Most striking is the absence of data for the first period in articles with a general, both positive and negative connotation. Comparing the previous data, it is most distinct at this point that the articles convey either entirely negative or positive connotation. The comprehensive connotations of articles are largely present in describing events abroad and almost non-existent regarding events occurring at the same time in abroad and in Slovenia. More than a half of the articles from the first period, describing events in Slovenia and almost a third of events abroad, convey a positive connotation.

41 Stuart Hall et al. Representation, The Open University SAGE, London, 2013, $10-11$.

42 In the second period during cross-referencing of the variables of location and connotation, "chi-quadrato" exceeds the value of 0,05 (value 0,2 ), therefore the data of the 2 nd period do not provide statistical representativeness. 


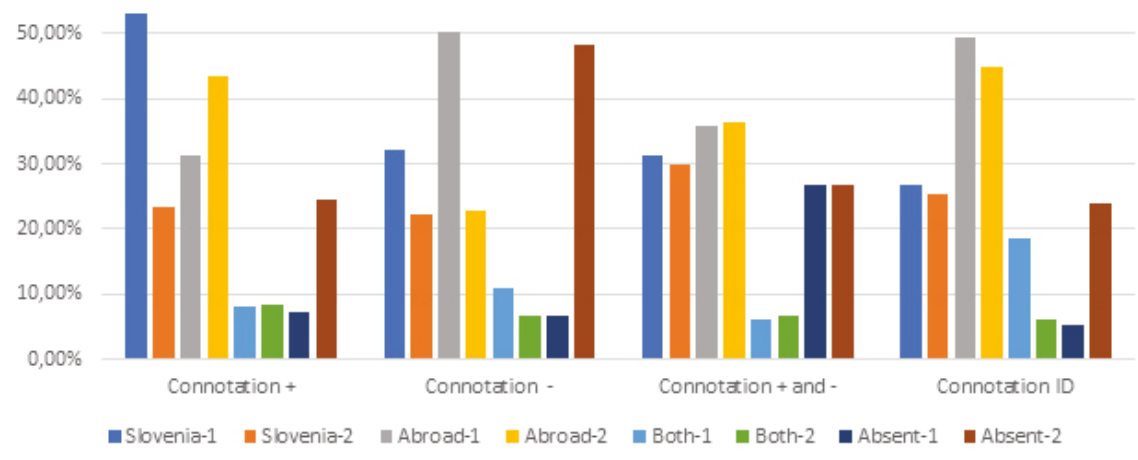

Graph 2. Connotation of articles according to location in the 1st and 2nd periods

In the first period, the highest number of articles depicting events related to the Church in Slovenia carry a positive connotation, followed by articles with negative connotation and a general connotation. The lowest number of articles convey ideological connotation (Table 9. Connotation of articles according to location in the 1st period). Church-related events abroad have a positive connotation (a third) and negative connotation (a third). Articles depicting events in the Church in Slovenia and abroad at the same time are also onethird negative and one-third positive. The absence of the location covers the largest share in articles with a general connotation.

Table 9. Connotation of articles according to location in the 1st period

\begin{tabular}{lccccc}
\hline Period 1 & Slovenia & Abroad & Both & Absent & Total \\
Connotation + & 254 & 150 & 39 & 34 & 477 \\
& $51,80 \%$ & $30,30 \%$ & $31,70 \%$ & $24,60 \%$ & $38,30 \%$ \\
Connotation - & 103 & 161 & 35 & 21 & 320 \\
& $21,00 \%$ & $32,50 \%$ & $28,50 \%$ & $15,20 \%$ & $25,70 \%$ \\
$\begin{array}{l}\text { Connotation }+ \\
\text { and - }\end{array}$ & 87 & 99 & 17 & 74 & 277 \\
& $17,80 \%$ & $20,00 \%$ & $13,80 \%$ & $53,60 \%$ & $22,20 \%$ \\
Connotation & 46 & 85 & 32 & 9 & 172 \\
ID & $9,40 \%$ & $17,20 \%$ & $26,00 \%$ & $6,50 \%$ & $13,80 \%$ \\
& 490 & 495 & 123 & 138 & 1246 \\
Total & $100,00 \%$ & $100,00 \%$ & $100,00 \%$ & $100,00 \%$ & $100,00 \%$ \\
\hline
\end{tabular}


David Kraner, Intolerance in the Media and Representations of the Catholic Church

Table 10. Connotation of articles according to location in the 2nd period

\begin{tabular}{lccccc}
\hline Period 2 & Slovenia & Abroad & Both & Absent & Total \\
Connotation + & 42 & 78 & 15 & 44 & 179 \\
& $33,10 \%$ & $42,40 \%$ & $40,50 \%$ & $26,20 \%$ & $34,70 \%$ \\
Connotation - & 37 & 38 & 11 & 80 & 166 \\
& $29,10 \%$ & $20,70 \%$ & $29,70 \%$ & $47,60 \%$ & $32,20 \%$ \\
Connotation + & 31 & 38 & 7 & 28 & 104 \\
and - & $24,40 \%$ & $20,70 \%$ & $18,90 \%$ & $16,70 \%$ & $20,20 \%$ \\
\multicolumn{7}{c}{ Connotation ID } & 17 & 30 & 4 & 16 & 67 \\
Total & $13,40 \%$ & $16,30 \%$ & $10,80 \%$ & $9,50 \%$ & $13,00 \%$ \\
& 127 & 184 & 37 & 168 & 516 \\
& $100,00 \%$ & $100,00 \%$ & $100,00 \%$ & $100,00 \%$ & $100,00 \%$ \\
\hline
\end{tabular}

In the second period, the highest number of articles depicting events related to the Church in Slovenia is polarized: a third with positive connotation and a third with negative connotation (Table 10. Connotation of articles according to location in the 2nd period). Events related to Church abroad convey mostly a positive connotation. Equally, it applies to events related to the Church abroad and in Slovenia. Articles about the Church without any information regarding the location of events convey mostly negative connotation.

To summarize, in the analysis of location of event and connotation, articles with a positive connotation stand out the most: in the first period, events depicting the Church in Slovenia, and in the second, events abroad. The positive connotation in the articles immediately follows the negative. The polarization of the positive and negative connotations is observed in events in Slovenia in the second period and abroad in the first period. Where the location of the event is absent, articles mostly caonvey negative or a general connotation. In short, the connotation of the articles changes from period to period regardless of the place of occurrence.

\subsection{Connotations and topic of articles}

In an environment where the media strives to survive, aims to please, mainly negative and ideological content is reported, thus setting aside the basic task of journalism, which is to provide truth, 
credible information and plurality of topics. ${ }^{43}$ We were interested in how the authors of the articles presented a certain topic in connection with the Catholic Church, assuming that articles with a positive connotation on various topics form positive representations of the Catholic Church (CC) and negative connotations negative. We strived to determine this by crossing-checking the basic topic of the article with the connotation of the article. This analysis contains only data from the last three periods. The highest number of articles with positive connotations is on charity, celebration, art, renovation, and others. The negative connotation is mostly on affairs, money, sexual abuse, history, post-war massacres, and politics. The ideological connotation is mostly on hate speech, terrorism, religions, after war massacres, terrorism and migrants. In the second period, hate speech is present in a third of the articles with positive connotation, the rest with ideological connotation, and in the third and fourth periods, more than half the articles with ideological connotation, while the rest are equally distributed between articles with positive and negative connotations. The ideological connotation of hate speech is therefore a problem in the Slovenian media, which are complicit in Christianophobia.

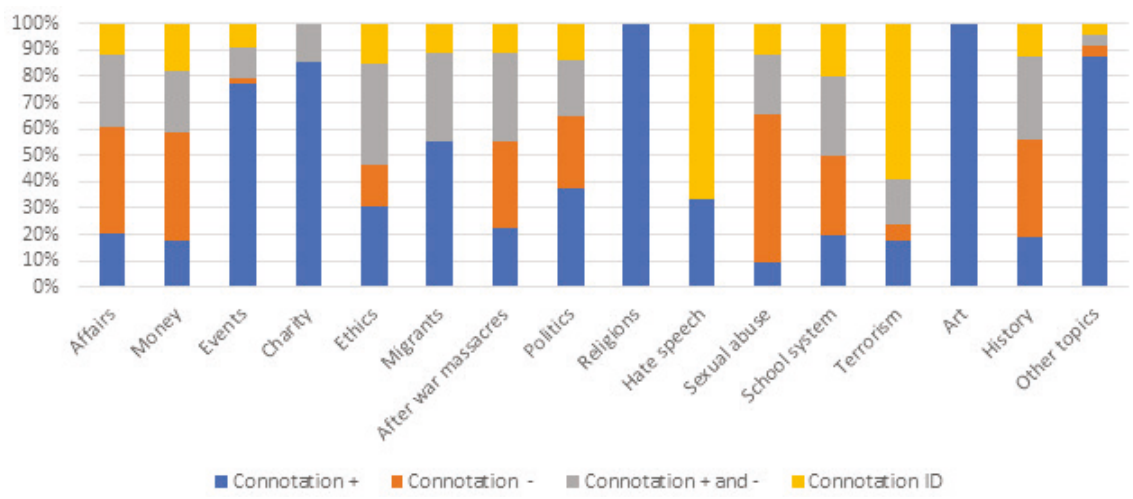

Graph 3. Connotations according to topic of articles in the 2nd period.

43 Bill Kovach - Tom Rosenstiel, I fondamenti del giornalismo. Ciò che i giornalisti dovrebbero sapere e il pubblico dovrebbe esigere, Dada Effe, Torino, 2007, 21. 


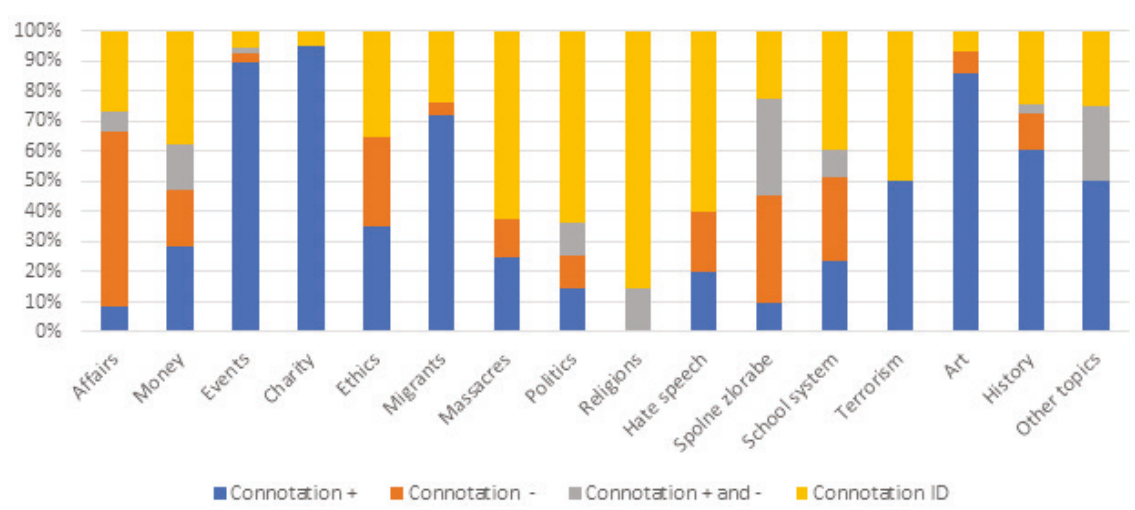

Graph 4. Connotations according to topic of articles in the 3rd period.

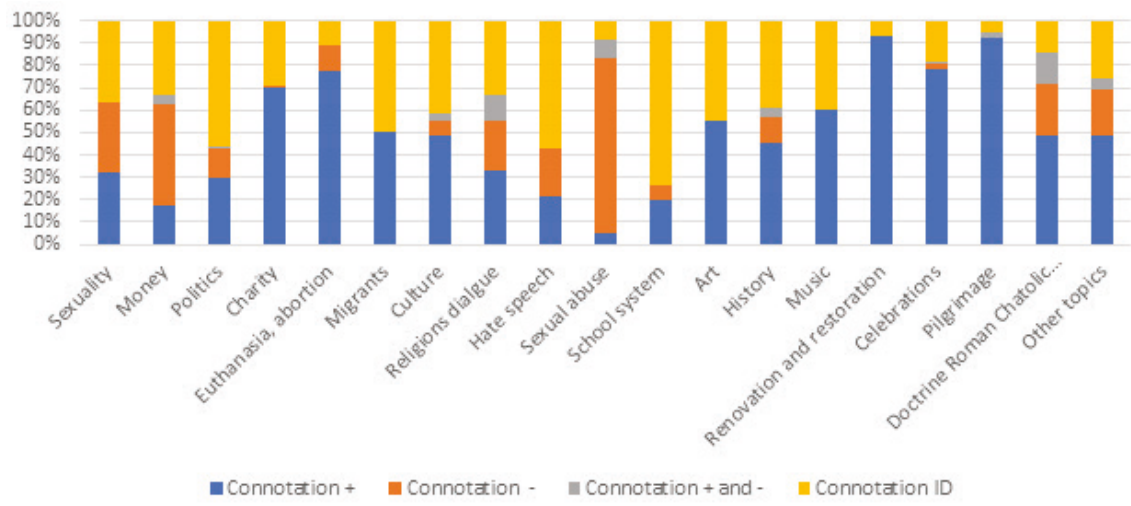

Graph 5. Connotations according to topic of articles in the 4th period.

To sum up, the authors of the articles write positively on not so serious socio-political and living issues; however, they cannot avoid doing so with topics such as: charity, celebrations, art, building renovations and other topics. Regarding serious issues such as money, sexual abuse, history, after war massacres, various scandals and politics, their articles usually carry negative connotations without a crumb of the positive - the problem of comprehensive reporting. These include problems in the areas of school system, paying taxes, real estate, spiritual care in hospitals, protection of minors, sexual abuse, etc. The ideological connotation is mostly present in topics on hate speech, terrorism, religions, after war massacres, terrorism and migrants. The topic of hate speech in particular is unreasonably ideologically present in the Slovenian media. 


\section{Findings AND CHALLENGES FOR THE FUTURE}

The presented analysis on media reporting is a challenge for a joint dialogue between the Slovenian media and the Catholic Church in Slovenia. Unity cannot be expected in everything, as there will always be tensions between the media and the Church.

The findings can be summarized in seven points: 1) authors, 2) topics, 3) connotations of articles, 4) media and the connotations of articles; 5) presence of photographs, 6) connotations and location, 7) connotations and topic. 1) The study highlights the problems of the six most negative authors. The solution could be to address the authors on a personal level. This could be an endeavour for a spokesperson of the Slovenian Bishops' Conference (SBC) to meet each journalist separately and try to reach a consensus. An informal meeting with each of them would allow them to discuss the offensive, unacceptable elements of Christianophobia and to hear the reasons for such articles. 2) The most attractive topics for the media in which the Church is most negatively represented are sexual abuse, money and politics. These three topics are the most sensitive and must be professionally resolved within the Church of Slovenia professionally: leave all sexual abuse to be investigated by a third party, punish criminals and compensate for the damage; finances need to be fully transparent; only experts or a newly established professional Catholic lobby group can actively be involved in socio-political issues. These topics should be the subject of a wider discussion at the Faculty of Theology, among priests and among bishops, and therefore it would be necessary to hold a press conference at the end of such seminars. 3) In contrast to almost half of the articles with negative and ideological connotation, which largely perpetuate negative attitudes towards the Church, it is necessary to look for the events in the Church that refute the above mentioned negative topics and systematically promote them in the media with sponsored articles. 4) Given the finding that the local media write articles mostly with a positive connotation, it is necessary to maintain positive contacts with them as well as hold informal meetings; as for the national media, we should consider whether to lease advertising space and thus promote positive events of the Church. 5) As the media that reports most negatively on the Church do not, as a rule, provide articles with photographs, it is necessary to send 1-2 photographs, in addition to public statements. 6) Since the connotations and location fluctuate from period to period, it is necessary to connect events in the Slovene Church whenever possible with world events and publish them on the SBC website. 7) The results of 
the connotations and topics indicate that the serious socio-political aspects of the Church are neglected in the media. This shows poor commitment on the part of the Church, which must not be content to make a public statement on behalf of the Justice and Peace Commission once a month. This must be planned, regular and connected with the Faculty of Theology and other professional organizations.

From the material presented, it is evident that Slovenian media play a role in spreading Christianophobia. The Catholic Church is expected to engage and strengthen communication in the future, which will actively reach out to journalists. The fact that the activity of the Church and that of the media are often on opposite sides must never be a reason for the spread of Christianophobia. It is a challenge for both parties to invest more effort, professionalism and also material resources to improve their mutual communication. In the absence of these changes, the number of conflicts will escalate and Christianophobia will spread.

\section{References}

Ameli, Saied R. - Syed M. Marandi - Sameera Ahmed - Seyfeddin Kara - And Arzu Merali, The British Media and Muslim Representation: The Ideology of Demonisatio. Islamic human Rights commission, Wembley, 2007.

Bajec, Anton et. al., Slovar slovenskega knjižnega jezika, Založba ZRC, Ljubljana, 2000.

Baker, Paul - Costas Gabrielatos - Tony McEnery, Discourse Analysis and Media Attitude. The Representation of Islam in the British Press, Cambridge University Press, Cambridge, 2013.

Barbano, Alessandro, Manuale di giornalismo in collaborazione con Vincenzo Sassu, Laterza, 2012, Roma-Bari.

Burow, Olaf-Axel, Was ist Gestaltpädagogik, in: Gestaltpädagogik in der Schule, Olaf-Axel - Herbert Gudjons (edd.), Bergamann und Helbig, Hamburg, 1998.

Castells, Manuel, Comunicazione e Potere, Università Bocconi Editore, Milano, 2009.

Dulles, Avery, Religion and the News Media: A Theologian Reflects, in: America, 9 (1994), 6-9.

Fantini, Ellen, Report 2018, Observatory on Intolerance and Discrimination against Christians in Europe, Vienna, 2018.

Fantini, Ellen, Report 2019, Observatory on Intolerance and Discrimination against Christians in Europe, Vienna, 2019.

Galli, Ida, La teoria delle rappresentazioni sociali, Il Mulino, Bologna, 2006.

Grausgruber, Heinrich, Gestalt-integrative Fortbildung der Religionslehrerinnen. Doctor dissertation at Kath, Theologischen Hochschule Linz, 1984. 
Hall, Stuart - Jessica Evans - Sean Nixon, Representation, The Open University SAGE, London, 2013.

Hrvatin, Sandra B. - Brankica Petković, In temu pravite medijski trg? Vloga države $v$ medijskem sektorju $v$ Sloveniji, Mirovni inštitut, Ljubljana, 2007.

Katz, Elihu - Paul F. Lazarsfeld, L'influenza personale in comunicazione, Armando, Rome, 2012.

Klaper, Joseph T, The Effects of Mass Communication, Free Press, Glencoe - Illinois, 1960.

Kovach, Bill - Tom Rosenstiel, I fondamenti del giornalismo. Ciò che i giornalisti dourebbero sapere e il pubblico dovrebbe esigere, Dada Effe, Torino, 2007.

Kraner, David, Le rappresentazioni sociali della Chiesa Cattolica in Slovenia, LAS, Rome, 2019.

Lazarsfeld, Paul - Bernard R. Berlson - William N. McPhee, Voting. A study of opinion formation in a presidential campaign, University of Chicago Press, Chicago, 1954.

Lah, Peter, Laži, preoblječne v novice, da ljudje dobimo norice, February 15 2018, www.academia.edu/35927042/Fake_news_Laži_preoblečene_v_ novice_ki_povzročijo_da_ljudje_dobimo_norice (acquired September 9 2020).

Lazarsfeld, Paul - Bernard R. Berlson - William N. McPhee, Voting. A study of opinion formation in a presidential campaign, University of Chicago Press, Chicago, 1954.

Lippmann, Walter, Public Opinion, Harcourt - Brace, New York, 1922.

Losito, Gianni, Il potere dei madia, Carocci, Rome, 2007.

Losito, Gianni, Il potere del pubblico. La fruizione dei mezzi di comunicazione di massa, Carocci, Rome, 2003.

Lukas, Elisabeth, Izviri, ki napajajo življenje s smislom, Novi svet, Ljubljana, 2015.

McQuail, Denis, Mass Communicatiion Theory an introduction, Sage, London, 1983.

Moscovici, Serge, La psychanalyse son image et son public, Presses Universitaires de France, Paris, 1975.

Moscovici, Serge, Sta arrivando l'era delle rappresentazioni, in: Moscovici. La vita, il percorso intellettuale, i temi, le opere, Mirila Bonnes (ed.), Franco Angeli, Milan, 1999.

Moscovici, Serge, Préface, in: Santé et maladie. Analyse d'une représentation sociale, 7-12, $\mathrm{Cl}$ audine Herzlich (ed.), Éditions de l'EHEES, Paris, 2005.

Moscovici, Serge, La psicoanalisi. La sua immagine e il suo pubblico, Unicopli, Milan, 2011.

Moscovici, Serge, Le rappresentazioni sociali, Mulino, Bologna, 2013.

Neuhold, Hans, Arbeitsgemeinschaft der Institute für Integrative Gestaltpädagogik und Seelsorge, in: Integrative Gestaltpädagogik und Seelsorge, 28 (2002). 
Noelle-Neumann, Elisabeth, La spirale del silenzio. Per una teoria dell'opinione pubblica, MELTEMI, Rome, 2002.

P. A, Najbolj priljubljeni slovenski mediji v 2018: Nedeljski dnevnik, Radio 1, Pop TV and 24ur.com. (March 27 2019), https://moski.hudo.com/aktualno/najbolj-priljubljeni-slovenski-mediji-v-2018-nedeljski-dnevnik-radio-1-pop-tv-24ur-com/ (acquired September 9 2020).

Pratellesi, Marco, New Journalism. Teorie e tecniche del giornalismo multimediale, Bruno Mondadori, Milan, 2008.

Perls, Frederick, Das Ich, der Hunger und die Aggression, Klett-Cotta, Stuttgart, 1987.

Petković, Brankica - Marko Prpič - Neva Nahtigal - Sandra B. Hrvatin, Spremljanje in vrednotenje medijev. Anketa med študenti, etničnimi manjšinami in politiki $v$ Sloveniji, Mirovni inštitut, Ljubljana, 2009.

Pogliano, Andrea, Le immagini delle notizie. Sociologia del fotogiornalismo, Edizioni Unicopli, Milan, 2009.

Polner, Kovačič Melita, Novinarska (iz)virnost. Novinarji in njihovi viri v sodobni slovenski družbi, Fakulteta za družbene vede, Ljubljana, 2004.

Price, Vincent, L'opinione pubblica, Il Mulino, Bologna, 2004.

Russo, Massimo - Zambardino Vittorio, Eretici digitali. La rete è in pericolo, il giornalismo pure come salvarsi con un tradimento e 10 tesi, Apogeo, Milan, 2009.

Slovenska škofovska konferenca, Izredna navodila slovenskih škofov za preprečevanje širjenja COVID-19: odpoved svetih maš do preklica, (March 13 2020), https://katoliska-cerkev.si/izredna-navodila-slovenskih-skofov-za-preprecevanje-sirjenja-koronavirusa-covid-19-odpoved-svetih-mas-do-preklica (acquired March 13 2020).

Šuen, Matjaž, Preiskovalno novinarstvo, Fakulteta za družbene vede, Ljubljana, 1994.

Tajništvo Slovenske škofovske konference, Letno poročilo 2019, November 22 2019, https://katoliska-cerkev.si/media/datoteke/Dokumenti\%20 in\%20publikacije/LETNO\%20POROCILO\%202019-OBJAVA\%20 SPLET.pdf (acquired December 1 2019).

Ule, Mirjana, Socialna psihologija. Analitični pristop $k$ življenju $v$ družbi, Založba FDV, Ljubljana, 2009.

Winnicott, Donald Woods, Vom Spiel zur Kreativität, Klett-Cotta, Stuttgart, 1974.

Wolf, Mauro, Teorie delle comunicazioni di massa, Bompini, Milan, 1992.

Zanacchi, Adriano, Opinione pubblica, mass media, propaganda, Las, Rome, 2006. 